
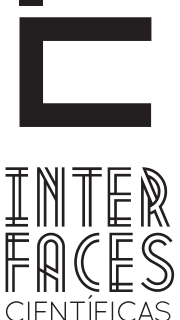

HUMANAS E SOCIAIS

\title{
CAPACIDAD COMPETITIVA DE LOS PRODUCTOS DE EXPORTACIÓN DE MADERA: APLICACIÓN PARA PEQUEÑAS Y MEDIANAS EMPRESAS DE MISIONES, ARGENTINA
}

Marina Guarrochena de Arjol $^{2}$
Amalia María Lucila Diaz ${ }^{2}$

\section{RESUMEN}

El estudio analiza el posicionamiento de productos en madera y sus manufacturas de las exportaciones de Argentina, ante cambios en la demanda internacional, durante el período 2006-2010. El enfoque está basado en el concepto retrospectivo de competitividad, ex-post, vinculada con el desempeño comercial y a incrementos de la cuota de mercado. Con la aplicación de una matriz de competitividad se pretende medir la capacidad competitiva de Argentina, adaptándose la estructura de sus exportaciones a la dinámica de la demanda internacional de la madera, a partir del desempeño de las exportaciones nacionales y de la identificación de productos de la oferta exportable de la provincia de Misiones. Este trabajo proporciona un marco instrumental de la competitividad sectorial, desagregado a nivel de productos, a través del análisis de sus capacidades para reconocer oportunidades en los sectores dinámicos e incrementar sus participaciones. Aporta una caracterización del comportamiento competitivo de los principales productos de exportación de las pequeñas y medianas empresas de Misiones, para examinar su potencial para la inversión y promoción del comercio y facilitar la apropiación práctica en los organismos regionales de apoyo exportador.

\section{PALABRAS CLAVE}

Exportaciones. Competitividad Ex Post. Desempeño Competitivo. Demanda Internacional. Matriz de Competitividad. Madera y sus Manufacturas. 


\section{ABSTRACT}

The study analyzes the positioning of wood products and manufactured exports in Argentina to changes in international demand for the period 2006-2010. The approach is based on the concept of competitiveness retrospective, ex-post, linked to business performance and increases in market share. With the application of an array of competition it is intended to measure the competitiveness of Argentina, adapting the structure of its exports to the dynamics of international demand of wood, from the development of national exports and identification of products that promote exports of State of Misiones. This paper provides a framework of instrumental competitive sector, disaggregated product level, through the analysis of their ability to recognize opportunities in the dynamic sectors as well as increase their holdings. It provides a characterization of the competitive behavior of the main export products of small and medium enterprises in Misiones, to examine their potential for reversing and promotion of trade and facilitate the ownership practice in regional bodies supporting exporter.

\section{KEYWORDS}

Exports. Ex-post Competitiveness. Competitive Performance. International Demand. Array of Competitiveness. Wood Products Manufactured.

\section{INTRODUCCIÓN}

Los continuos cambios en el comercial internacional, con la aparición de nuevos competidores y los acuerdos comerciales, justifican el replanteamiento de las acciones comerciales, tanto en el entorno empresarial como por parte de los organismos estatales encargados de su promoción.

\section{RESUMO}

0 estudo analisa o posicionamento de produtos em madeira e suas manufaturas das exportações de Argentina ante as mudanças na demanda internacional, durante 0 período 2006-2010. 0 enfoque está baseado no conceito restropectivo de competitividade, ex-post, vinculada com o desenvolvimento comercial e com incrementos da cota do mercado. Com a aplicação de uma matriz de concorrência pretende-se medir a capacidade competitiva da Argentina, adaptando a estrutura de suas exportações à dinâmica da demanda internacional da madeira, a partir do desenvolvimento das exportações nacionais e da identificação dos produtos da promoção que se exporta do Estado de Missiones. Este trabalho proporciona um marco instrumental da competitividade sectorial, desagregado a nível de produtos, através da análise de suas capacidades para reconhecer oportunidades nos setores dinâmicos, como também incrementar suas participações. Aporta uma caracterização do comportamento competitivo dos principais produtos de exportação das pequenas e médias empresas de Missiones, para examinar seu potencial para a inversão e promoção do comercio e facilitar a apropriação prática nos órgãos regionais de apoio exportador.

\section{PALAVRAS CHAVE}

Exportações. Competitividade Ex-post. Desenvolvimento competitivo. Demanda Internacional. Matriz de Competitividade. Madeira e suas Manufaturas.

El comercio exterior es una temática intensiva de información, y existe una amplia diversidad de servicios y herramientas para el análisis del comercio y la política comercial, sin embargo, las pequeñas y medianas empresas-PyMes, según Svarzman (2008, p. 60) son "las que se enfrentan con mayores dificul- 
tades y restricciones para acceder, seleccionar e interpretar la información que resulta relevante y estratégica para sus negocios".

La provincia de Misiones es el principal centro productor y exportador forestal de Argentina, siendo su oferta exportadora el $43 \%$ de madera y sus manufacturas; predominantemente constituida por PyMes que abastecen al mercado de productos de bosques implantados de coníferas.

La crisis de los préstamos hipotecarios en Estados Unidos, y consecuente crisis financiera mundial, modificó el escenario de expansión nacional de las ventas al mercado externo de los productos de madera, consecuencia del impacto sobre la construcción de viviendas en el país del Norte y con ello la disminución de demanda de una amplia variedad de productos de maderas. En este contexto, la actividad exportadora local direccionó parte de sus ventas hacia el mercado interno, limitando el compromiso de las empresas en el mercado mundial.

Desde la visión sectorial de la madera de Argentina, Rivello y Marecos (2009, p. 62) sostienen que el crecimiento sostenido a las PyMes depende de la generación de estrategias a largo plazo, la diversificación de productos y la apertura de mercados. La crisis provocó la retracción de la demanda de los países desarrollados, aunque no ha afectado a las principales economías con la misma intensidad, que con el avance de los mercados emergentes ha modificado el escenario mundial. La distinta estructura de las exportaciones de bienes en cada país, y la diferente evolución de la ventaja competitiva adquirida en los productos que comercializan, podría explicar estas diferencias.

En el marco conceptual del comercio internacional, Bonifaz y Mortimore (1999, p. 21) argumentan que los beneficios relativamente mayores son los países que logran incrementar su participación y/o concentrar sus exportaciones en sectores dinámicos, pues son los que presentan una rápida expansión en el comercio mundial. Al respecto existe una abundante literatura sobre la evolución de las exportaciones y la capacidad de una economía para competir en los mercados internacionales que evalúan como medida objetiva del resultado, los indicadores de las cuotas de mercado, denominada competitividad expost, asociada a la ampliación en la participación de los bienes de un país, región o empresas en los mercados internacionales, en un período determinado.

Ubfal (2004, p. 7) analiza que la comparación relativa de los niveles de eficiencia no se da sólo entre distintos países sino también entre los bienes de cada país, "De esta manera para aumentar las exportaciones de un bien, no sería suficiente que el mismo fuera competitivo internacionalmente, sino que debería ser seleccionado por las ventajas comparativas a nivel local".

Ante ello, el análisis se centra en el enfoque de la competitividad comercial ex-post de las exportaciones de Argentina, para valorar la evolución de la cuota-participación de los productos de madera e identificar el posicionamiento de productos de la oferta exportable de PyMes de Misiones. Esto permite examinar su potencial para la inversión y promoción del comercio para sostener o aumentar su presencia en los mercados internacionales, y facilita la apropiación práctica en los organismos regionales de apoyo exportador. 


\section{MÉTODO}

Este estudio se sustenta un análisis retrospectivo de la competitividad ex-post del desempeño comercial de Argentina en mercados internacionales y la capacidad competitiva de la oferta exportable de productos de madera y sus manufacturas, en el período 2006-2010.

Desde este enfoque, el estudio se basa en la participación relativa en el mercado internacional y el cambio de la misma en función de indicadores económicos basados en datos de los flujos comerciales, evaluándose la competitividad de un país a través del desempeño competitivo nacional en el comercio mundial y su capacidad de oferta con miras a especializarse en sectores más dinámicos. Este indicador de competitividad, de acuerdo a Bonifaz y Mortimore (1999, p. 29) no hace referencia a los factores explicativos que determinaron la competitividad alcanzada, y tampoco separa la competitividad lograda en bases de largo plazo de aquella fundada en condiciones efímeras.

Este estudio resulta de una investigación de la Universidad Nacional de Misiones sobre Oportunidades de negocios internacionales de la madera- Código No 16/E122, que analizó el comportamiento y tendencia del mercado internacional de la madera aserrada y sus manufacturas, y la capacidad de oferta exportable de las PyMes de Misiones, a fin de identificar mercados potenciales y diseñar estrategias de gestión para el seguimiento periódico de los mercados (Díaz et al., 2011).

Se aplica el modelo de desempeño comercial y competitividad internacional, de la base de datos de comercio de bienes del Centro de Comercio Internacional $(\mathrm{CCl}, 2012)$ que brinda acceso abierto a diferentes herramientas de interpretación de indicadores económicos y de análisis de mercado, en base a estadísticas comerciales de Naciones Unidas -UN
COMTRADE, con productos definidos de la rama madera y sus manufacturas, a un nivel de 4, 6 y 10 dígitos, según la nomenclatura internacional del Sistema Armonizado de Designación de Mercaderías.

Para examinar la capacidad de adaptación de la oferta nacional a la demanda internacional, la base de datos de $\mathrm{CCl}$ (2009, p. 50-57) proporciona una matriz de competitividad que facilita interpretar la competitividad de un país a través del análisis de sus capacidades para reconocer oportunidades en los mercados dinámicos e incrementar sus participaciones.

Describe el posicionamiento de los productos en los mercados internacionales confrontando el crecimiento de la oferta nacional con el resultado de comparar la variación de la demanda internacional del producto con el crecimiento de importaciones del rubro al que pertenece el mismo.

La herramienta de análisis es una matriz de evaluación del comercio nacional desde la perspectiva de los productos, con esquemas simples de dos ejes y posicionamientos en cada cuadrante, en función del crecimiento anual de la participación de Argentina en las exportaciones mundiales en $\%$ y crecimiento anual de las importaciones mundiales, en \%, en el período 2006-2010.

Los indicadores de la evolución del crecimiento basados en los últimos 5 años, disponibles en el análisis de mercados de $\mathrm{CCl}$, se calculan utilizando el método de la tendencia de los mínimos cuadrados logarítmicos, en serie de valores expresados en dólares con datos de UN COMTRADE.

Los criterios de análisis del modelo del $\mathrm{CCl}$, para clasificar los productos de exportación están basados en la interpretación del comportamiento de la tasa media anual de crecimiento nominal de: 
- Las exportaciones del rubro o producto del país respecto a la tasa de crecimiento de las importaciones mundiales del producto, donde el incremento anual de la cuota de exportaciones en el mercado mundial representa el "Crecimiento anual exportaciones en valor entre 2006-10" menos la "media de crecimiento anual de las importaciones mundiales entre 2006-10 del producto".

- de las importaciones mundiales del producto respecto a la tasa de crecimiento de las importaciones mundiales totales.

Así, en un país, cuando las exportaciones del producto ha crecido más rápido que las importaciones mundiales del producto, por lo tanto se trata de un país competitivo que gana participación en un mercado en expansión y atractivo. Y si las exportaciones del producto crecen menos que las importaciones mundiales del producto, el país no está aprovechando el potencial de expansión del mercado mundial del producto y está perdiendo participación en el mercado mundial .
En el mismo sentido, se puede estar ganando o perdiendo participación en un mercado mundial de un producto que está creciendo por encima o por debajo de lo que crece el total de importaciones mundiales.

En el caso de un producto cuya tasa de crecimiento de las importaciones mundiales es mayor que la tasa de crecimiento de las importaciones mundiales totales, es un mercado en desarrollo y atractivo por encima del promedio mundial. Si la tasa de crecimiento es menor a la mundial se trata de un mercado que crece poco en relación al comercio mundial. Y si la tasa de crecimiento es negativa es un mercado en declive.

A partir de este razonamiento, surgen cuatro categorías de posicionamiento de los productos, como puede ser observado en Figura 1. La clasificación de las posiciones de productos de exportación, es orientativa útil en el primer paso analítico. Para las aplicaciones concretas de las políticas y estrategias para la promoción comercial de un producto específico se debe profundizar el enfoque y considerar otros aspectos comerciales.

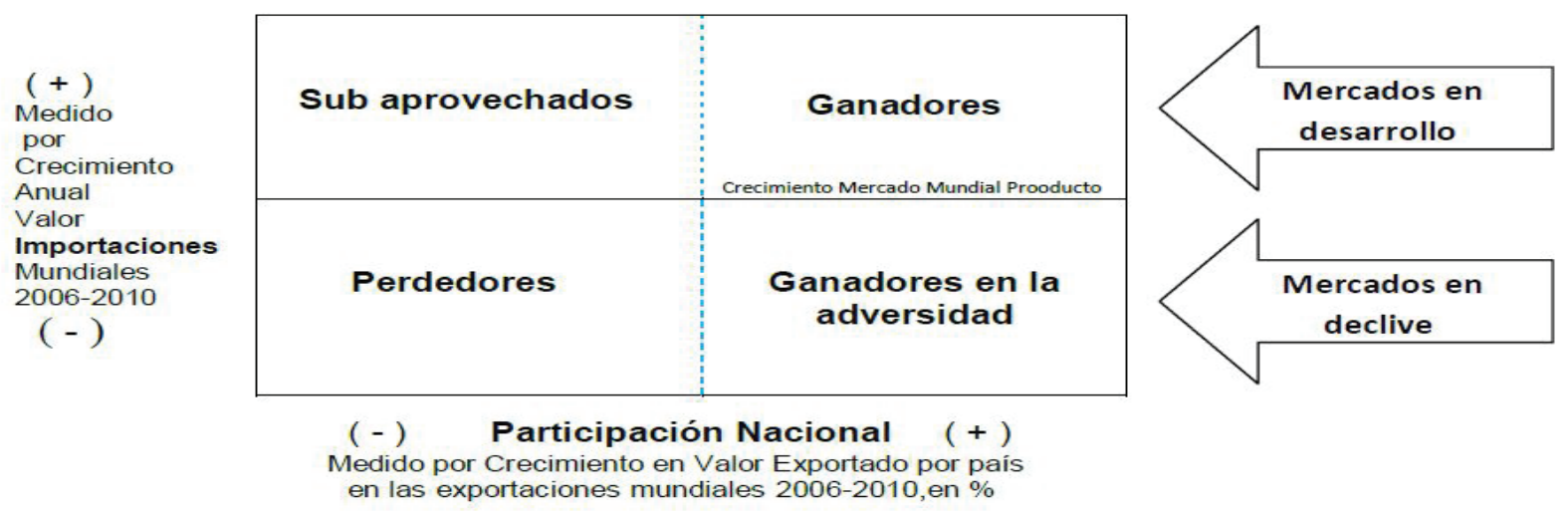

Figura 1: Matriz de competitividad de productos exportados Fuente: Elaboración propia en base a Cálculos del $\mathrm{CCl}$ 
Ganadores en los sectores en desarrollo (Champion): En el cuadrante superior derecho contiene ganadores en sectores en crecimiento, se trata de productos de exportación de alto crecimiento de la cartera de productos del país y una demanda mundial por encima de la tasa promedio mundial. Comprenden productos, que están creciendo más rápido que el comercio mundial en general, y el país ha sido capaz de superar el crecimiento del mercado mundial y tiene, por consiguiente, el aumento de su participación en las exportaciones mundiales.

Perdedores en los sectores en desarrollo (oportunidades perdidas): Situado en el cuadrante superior izquierdo, se trata de sectores de bajo rendimiento en los sectores en expansión, el país ha perdido cuota de mercado mundial, mientras que el mercado mundial ha crecido. .

Ganadores en sectores en declive: Los mercados mundiales de importación de productos de este cuadrante inferior derecho, están creciendo por debajo de la tasa promedio mundial, pero la cuota de mercado de los exportadores del país es cada vez mayor.

Perdedores en sectores en declive: Las importaciones mundiales del producto se han incrementado a una tasa inferior a la media y la participación del país en el mercado mundial ha disminuido.

\section{EL COMPORTAMIENTO DE PRODUCTOS}

Las exportaciones de madera y sus manufacturas en el comercio mundial alcanzaron a 106.150 millones de dólares para el año 2010, siendo el principal rubro de producto exportado madera aserrada con el $27,20 \%$, que junto a la madera en bruto, contrachapada, obras y piezas de carpintería para construcción, tableros de fibra y de partículas, concentran las tres cuarta partes del comercio.

El complejo exportador de origen forestal en Argentina representa el $1,50 \%$ de las ventas argentinas al exterior al 2010, según el Ministerio de Economía de Argentina (2011, p. 2), constituido por productos del complejo celulósico papelero (65\%) y maderero (24\%); y la mayor participación proviene de las provincias de Misiones (27\%) y Buenos Aires (24\%).

La Argentina se caracteriza por ser tradicionalmente un país con balanza comercial deficitaria en el comercio de productos forestales, de 571 millones de dólares. Sin embargo, las ventas al exterior en el rubro de maderas y sus manufacturas registran 274,5

millones de dólares, con una balanza comercial positiva, de 136,7 millones, para el año 2010.

En un contexto de retracción del flujo del comercio internacional, las exportaciones nacionales registraron un crecimiento anual promedio del $11 \%$ en el período 2006-2010, con los valores más altos de la serie en el último año. Este impacto de la crisis tuvo repercusiones desiguales en las distintas economías del mundo.

En cuanto a la exportación de productos específicos del rubro madera y manufacturas,, ocho productos tienen una participación del $90,10 \%$ y el restante está representado por 23 productos de significación en el comercio mundial. El $80 \%$ de la oferta de bienes de origen maderero exportable están concentrados en: tableros de fibra, en la posición arancelaria441114 representa el $23,80 \%$, madera aserrada de coníferas- 440710 el 16,72\%, carbón vegetal- 440290 el $16,3 \%$, y los tableros de partículas - 441011 el $12,80 \%$. 
La provincia de Misiones participa con el 42,90\% de las exportaciones totales de Argentina en madera y sus manufacturas, por un valor de 117,7 millones de dólares, en el año 2010. En la Figura 2, se observa que la contribución más importante a las exportaciones argentinas son los productos Madera aserrada de coníferas, tableros de fibra y de partículas, madera perfilada de coníferas, y en menor valor relativo las demás manufacturas de madera, herramientas y mangos de madera, contra chapado, hojas para chapado, aserrín y carpintería para construcción.

Según el estudio de la Universidad Nacional de Misiones (Diaz, et al, 2011) la crisis de las hipotecas en Estados Unidos impactó sobre las exportaciones de productos de madera de pino, siendo la madera de conífera el componente principal de los productos de madera y sus manufacturas exportados por Misiones, restringiendo los volúmenes exportados en un $32,80 \%$ en el período $2006-2010$ y un incremento

\section{DESEMPEÑO COMPETITIVO DE ARGENTINA}

En este contexto, se analiza desempeño competitivo de los productos exportados por Argentina, de acuerdo a la matriz proporcionada por ITC, en función de la oferta de Argentina y la demanda internacional, en el período 2006 - 2010,

Se describe, en general, la posición de la cartera de los productos identificados en la Figura 3, y el potencial de inversión y promoción; y en particular el comportamiento de la demanda internacional de algunos productos significativos de la oferta exportable de Misiones.

El sector de ganadores en los mercados en desarrollo incluye productos particularmente dinámicos, donde Argentina ha obtenido buenos resultados, tableros de partículas -441039 , aserrín y desperdi- de los valores en dólares del 14\%, a partir del 2010, como consecuencia del aumento vía precios de los productos.

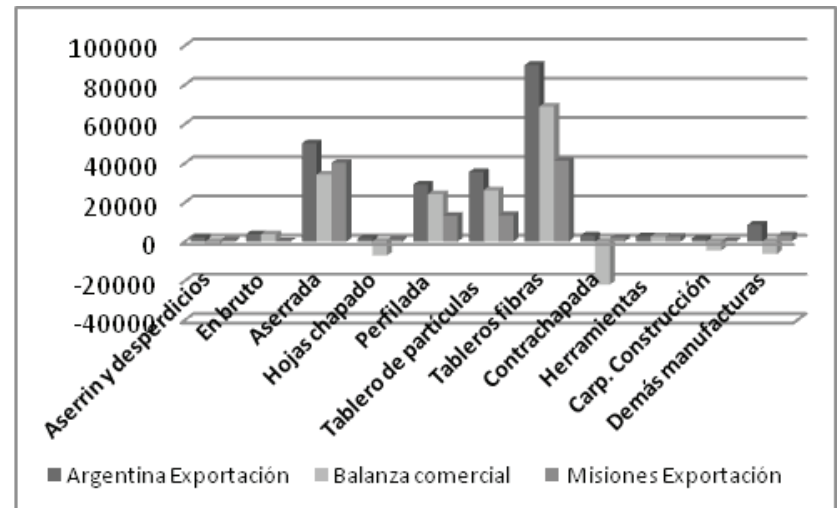

Figura 2: Exportaciones, en miles de dólares, 2010

Fuente: Elaboración propia en base a datos del Instituto Nacional de Estadística y Censo. cios -440130 carbón vegetal-440200, para los cuales el valor de la exportación mundial está creciendo y con los cuales los exportadores argentinos han aumentado su participación. Los exportadores de estos productos demostraron su competitividad internacional durante el período. Los esfuerzos de promoción comercial para estos productos son menos riesgosos ya que son exportaciones nacionales exitosas y la demanda internacional crece. El objetivo de los esfuerzos de promoción de estos productos deberían direccionarse hacia la ampliación de la capacidad de oferta.

En el sector perdedores en los mercados en desarrollo, si bien la demanda internacional de tableros de fibra demás 0,5g-441129 y demás contrachapada -441299, ha estado creciendo a un ritmo por enci- 
ma del promedio, Argentina se ha estado quedando atrás. Sus exportaciones han bajado y como resultado, la participación de Argentina en el mercado internacional ha bajado. Estos productos corresponden a sectores dinámicos en mercados que crecen y representan retos particulares para los esfuerzos de promoción comercial, porque pueden representar oportunidades para mejor provecho de la creciente demanda internacional.
En general, el obstáculo no es la demanda internacional, sino más bien razones de bajo rendimiento y competitividad, e incluyen limitaciones en la capacidad de oferta, cuestiones de calidad del producto y las barreras de acceso al mercado. Para estos productos es esencial identificar y eliminar los obstáculos específicos que impiden una expansión más dinámica de la exportación.

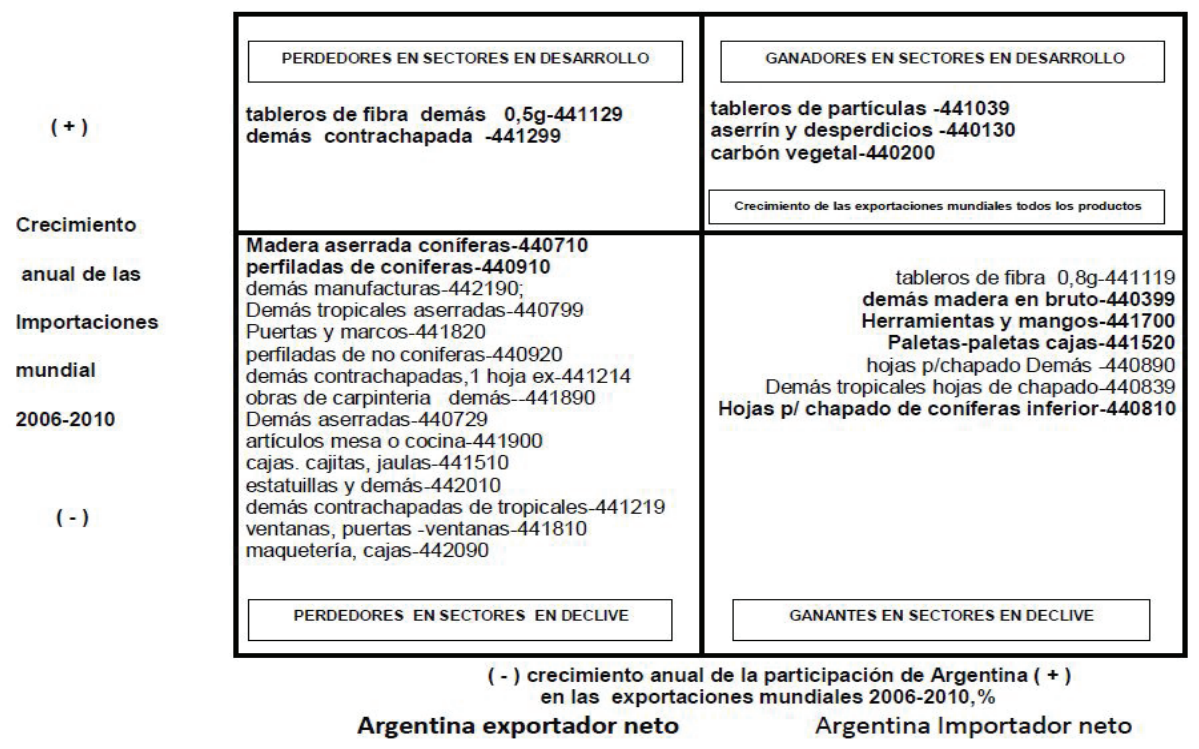

Figura 3: Matriz de competitividad de productos exportados por Argentina Fuente: Elaboración propia en base a Cálculos del CCl

Ganadores en los mercados en declive: Los mercados mundiales de importación para los productos tableros de fibra 0,8g -441119, Herramientas y mangos-441700, hojas chapado Demás -440890, Hojas chapado de coníferas inferior-440810, entre otros, están disminuyendo o creciendo por debajo de la tasa promedio mundial, pero la cuota de mercado de los exportadores de Argentina es cada vez mayor. Las exportaciones crecen a buen ritmo, con desempeño comercial positivo, pero en un sector con menor dinamismo que el comercio mundial, en declive o estancados (ga- nadores en la adversidad). Desde la perspectiva de la promoción comercial, se requeriría de estrategias para el marketing de nichos para aislar el desempeño comercial positivo del declive general en estos mercados.

El mercado de los tableros de fibra, representa uno de los principales productos que demandan los mercados mundiales, su tasa de crecimiento en el período 2006-2010 ha sido negativa(-1\%), pero su magnitud da cuenta que tuvo un impacto menor la crisis, para recuperar su crecimiento en 2010. 
Los tableros de fibra -441019 , que incluyen la posición 441014, representan el 29,50\% de dicho mercado, han disminuido su tasa de crecimiento en un $6 \%$ en valor con una leve recuperación del al 2010, pero ha aumentado las cantidades importadas. Lo que refleja un aumento de participación en las importaciones mundiales. Estos productos incluyen los tableros de fibra con mayor participación en las exportaciones argentinas y de Misiones.

Perdedores en los mercados en declive: La importación mundial de Madera aserrada coníferas-440710; perfiladas de coniferas-440910, demás manufacturas-442190; Puertas y marcos-441820: obras de carpintería, demás--441890; ventanas, puertas -ventanas-441810, entre otras ha incrementado a un ritmo por debajo del promedio o, en efecto, ha disminuido, y la participación del mercado de Argentina ha disminuido o mantuvieron estacionario su cuota de mercado. La caída de las exportaciones está asociado a un bajo rendimiento o desempeño exportador que supone ser desplazado en mercados de gran crecimiento, de crecimiento relativo o en mercados que incluso están en declive a nivel mundial.

Las perspectivas de exportación para estos productos tienden a ser poco alentadoras para Argentina. Los esfuerzos de promoción para los productos en esta categoría encaran una dificultad, se debería adoptar un enfoque integrado para tomar en cuenta los obstáculos tanto por parte de la oferta como de la demanda.

La importación mundial de la Madera aserrada de coníferas creció hasta el año 2007 para luego entrar en una fase recesiva, ante el impacto negativo de la crisis financiera mundial. El volumen de importaciones disminuyó un $7 \%$ en valores y del $5 \%$ en cantidades, pero en 2010 el mercado ha comenzado a presentar signos de recuperación, no alcanzando los valores del inicio de la crisis.

Las tasas de crecimiento promedio de las importaciones mundiales de la Madera perfilada de coníferas decrecieron en valores en un $-8 \%$ y en cantidades el $-11 \%$.

La madera contrachapada es uno de los principales productos de importación, el crecimiento anual ha disminuido (6\%) pero sus cantidades importadas aumentaron un $11 \%$, ganando participación de algunos productos en el comercio mundial.

La importancia del subsector de Obras y piezas de carpintería para construcciones, radica en el uso para la construcción e incluyen productos diversos manufacturados; ha crecido por debajo del comercio mundial, a tasa anuales de $-4 \%$, sin embargo algunos productos han aumentado su participación en la importaciones mundiales.

La mayor participación es de los productos de las demás obras y piezas de carpintería, con el 40,70\% de los valores importados en este subsector. La tasa de crecimiento de este producto decreció $(-4 \%)$ en el período en estudio, recuperando un $20 \%$ al 2010 , pero la demanda crece más rápidamente que las exportaciones mundiales.

Este rubro incluyen los productos Postes y vigas (vigas sólidas y multi laminadas) con un desempeño más dinámico en el comercio mundial, porque la demanda mundial creció en promedio anual un $28,66 \%$, en el período 2007-2010, más rápido que las exportaciones mundiales, y aumentó su participación en las importaciones mundiales en un $4 \%$. Por lo que, es producto atractivo en el mercado mundial para analizar la ampliación de la capacidad de oferta del producto.

Con respecto a la demanda mundial de puertas y sus marcos, aún con decrecimiento de su participación en el mercado importador mundial, este producto ha logrado aumentar su crecimiento en pequeños mercados.

El producto ventanas, puertas y ventanas, que representa un valor del $25,10 \%$ del subsector; ha au- 
mentado su participación en las importaciones mundiales, pero con tasas de crecimiento negativas(-1\%). Este producto a diferencia de los anteriores resalta, en general, tasas negativas (-6\%) al 2010, en los más importantes mercados.

Las Demás manufacturas, incluye los productos de madera que no han sido clasificado en las demás posiciones arancelarias, integrada por los tableros alistonados, con una tasa de crecimiento anual del $-2 \%$, y al 2010, luego de la crisis, registra una recuperación de los mercados (8\%).

En particular, en Argentina la participación en el comercio mundial de productos de madera en mercados en desarrollo ha presentado un comportamiento relativamente favorable en el periodo 2006-2010, coincidente con la expansión de las exportaciones en productos derivados de tableros de partículas y de fibra, indicativa de la tendencia del uso de este tipo de productos. En menor volumen rubros de los productos derivados de aserrín y madera contrachapada, que son producidos y exportados por las PyMes de Misiones.

En este caso, los productos de mayor expansión en la producción y exportación son la madera aserrada de coníferas y re manufacturas como las maderas perfiladas, que son gravitantes por el volumen de la demanda internacional, pero en mercados en declive o estancados. Estos productos están caracterizados por la formación de precios "comoditizados" en los mercados internacionales, y relativamente alejados geográficamente de los centros de producción final, que requieren esfuerzos desde la perspectiva de la promoción comercial y estrategias por parte de la oferta y la demanda.

\section{CONCLUSIÓN}

El mercado internacional de maderas es muy sensible a la crisis económica internacional, por la desaceleración que sufrió la industria de la construcción, aunque no ha afectado a las principales economías con la misma intensidad, ha modificado el escenario mundial, aumentando las presiones competitivas a las que se enfrentan las pequeñas y medianas empresas.

La estructura de especialización de las exportaciones de Pymes de Misiones, sesgada hacia productos de menor contenido de valor agregado y demanda menos dinámica, habría limitado avances en la participación de mercado en el período en estudio. Sin embargo, algunos productos específicos derivados de carpintería para la construcción, de poco volumen de producción, son más dinámicos, para expandir la oferta o identificar nuevos nichos de mercado.
Los productos con ventajas competitivas en el mercado internacional, están vinculados a mayores contenidos tecnológicos, como los derivados de tableros de fibra y madera con fines energéticos que favorecerían la industrialización de componentes para la generación de productos de mayor valor agregado y ganancias de calidad y de los procesos, dando respuesta a las necesidades de la industria del mueble y a una mejora de la competitividad. Por lo que, el desafío es desarrollar y diversificar los productos con una demanda más dinámica y con potencial de crecimiento en las empresas exportadoras.

Las investigaciones futuras podrían incorporar el factor distancia a los mercados de destino en el análisis de la capacidad exportadora, los costos de transporte pueden diferenciar las ganancias de competitividad-precio en las PyMes exportadoras de Misiones. 
Asimismo, este trabajo proporciona un marco instrumental de análisis sectorial de la competitividad, desagregado a nivel de productos, posibilitando la apropiación práctica del conocimiento en los organismos regionales de apoyo exportador; y permi- tiría realizar análisis de escenarios prospectivos de la demanda internacional con el seguimiento de los desempeños competitivos de los mercados, para reconocer oportunidades de inversión y de promoción del comercio.

\section{REFEREENCIAS}

ARGENTINA. Ministerio de Economía. Complejos Exportadores Provinciales. MECON, Buenos Aires, abr. 2011.

BONIFAZ, José Luis; MORTIMORE, Michael. Colombia: un CANálisis de su competitividad internacional. Santiago de Chile: CEPAL, 1999. (Serie Desarrollo Productivo, 58).

Centro de Comercio Internacional. Trade Map-User Guide: Trade statistics for international business development. Market analysis and research. Geneva: CCl, 2009. Disponible en: <http://legacy.intracen.org/marketanalysis/Docs/Trademap/TradeMap-Userguide-EN.pdf > Consultado el: 6 abr. 2012.

CCI. Trade Map: Estadísticas de comercio para el desarrollo de los negocios internacionales, Sección de Análisis de Mercados, 2012. Disponible en: < http://www.trademap.org>. Consultado el: 6 abr. 2012.

DÍAZ, Amalia María Lucila; et al. Oportunidades de negocios internacionales y Estrategias de seguimiento de los mercados de la madera aserrada y sus manufacturas de Misiones. Posadas: UNaM-FCE, 2011. Código de Investigación Nº 16/E122.

RIVELLO, Alejandro; MARECOS, Víctor. Desarrollo de nuevos mercados para los productos de madera argentina. Revista ASORA, Buenos Aires, Año 14, n. 83, may. /jun. 2009.

SVARZMAN, Gustavo. Gestión de información y servicios de apoyo a las empresas. En: INSTITUTO DE COMERCIO INTERNACIONAL. La internacionalización de empresas y el comercio exterior argentino. Buenos Aires: Fundación Standard Bank, 2008.

UBFAL, Diego. El concepto de competitividad. Medición y aplicación al caso Argentino. IIE-UBA, Buenos Aires, Documento de Trabajo n. 15, 2004. Disponible en: <http://www.funcex.org.br/material/REDEMERCOSUL_BIBLIOGRAFIA/biblioteca/ESTUDOS_ARGENTINA/ARG_42.pdf >. Consultado el: 23 feb. 2012. 

catedras de Metodologia de la investigación, Economía Aplicada. Investigado SPU categoría III. Facultad de ciencias Económicas, Unam-Ingeniera Agronoma, master en Administración estratégica, Unam, master en Integración Económica UIA, España.guarrochena@fce.unam.edu.ar

2 Profesora e Investigadora, Facultad de Ciencias Forestales, Universidad Nacional de Misiones, Profesora Adjunta dedicación excluiva catedra Administración y Economía forestal. Investigadora categoría III-SPU Ingeniera Forestal, Master en Economía Rural-UFV-Brasil. Idiaz@facfor. edu.unam.ar 